\title{
Plasma topiramate concentrations resulting from doses associated with neuroprotection against white matter injury and stroke in two strains of rat pups
}

\author{
Annie M. Clark ${ }^{1,2,7}$, John T. Mondick ${ }^{3}$, James C. Cloyd ${ }^{1,2}$, Athena F. Zuppa ${ }^{4}$, Yogendra H. Raol ${ }^{5}$ and Robert R. Clancy ${ }^{6}$
}

BACKGROUND: Cerebral white matter (WM) injury and stroke are common neuropathological injuries in newborns with congenital heart defects (CHDs) requiring surgery. Previous investigations in Long Evans rat pups subjected to hypoxia-ischemia found that intraperitoneal (i.p.) topiramate (TPM) at $30 \mathrm{mg} /$ $\mathrm{kg}$, but not $50 \mathrm{mg} / \mathrm{kg}$, conferred neuroprotection. In SpragueDawley pups, a dose of $30 \mathrm{mg} / \mathrm{kg}$ protected against stroke. Concentrations associated with neuroprotective doses were not measured. The aims of this investigation were to determine concentrations associated with neuroprotective doses and to investigate the pharmacokinetics (PK) of i.p. TPM.

METHODS: Concentration-time data following administration of 30 and $50 \mathrm{mg} / \mathrm{kg}$ doses were analyzed using nonlinear mixed-effect modeling.

RESULTS: Mean predicted steady-state maximum and average concentrations following $30 \mathrm{mg} / \mathrm{kg}$ TPM were 31.3 and $16.8 \mu \mathrm{g} / \mathrm{ml}$ in Long Evans and 39.9 and $24.4 \mu \mathrm{g} / \mathrm{ml}$ in SpragueDawley pups. Mean predicted steady-state maximum and average concentrations following $50 \mathrm{mg} / \mathrm{kg}$ TPM were 52.1 and $28.1 \mathrm{\mu g} / \mathrm{ml}$ in Long Evans and 66.5 and $40.6 \mu \mathrm{g} / \mathrm{ml}$ in SpragueDawley pups. The apparent clearance (CL/F) and apparent volume of distribution (V/F) were $0.0470 \mathrm{ml} / \mathrm{min}$ and $22.2 \mathrm{ml}$, respectively, for Long Evans and $0.0325 \mathrm{ml} / \mathrm{min}$ and $19.7 \mathrm{ml}$, respectively, for Sprague-Dawley pups.

CONCLUSION: TPM concentrations associated with neuroprotective doses were determined. Body size and strain were significant covariates on CL/F and V/F. Results provide targets for future neuroprotection studies.

$T$ he major neuropathological basis of lifelong disabilities in term infants born with serious forms of congenital heart defects (CHDs) is diffuse injury to the brain's white matter (WM), commonly called periventricular leukomalacia (PVL). PVL is found in more than $50 \%$ of magnetic resonance imaging examinations performed after infant heart surgery in term infants (1). Survivors of newborn heart surgery share a distinctive profile of chronic neurodevelopmental disabilities due to WM injury; the profile is characterized by attention deficit-hyperactivity disorder and impairments in cognition, executive function, speech, language, visual-spatial perception, and gross and fine motor abilities. The second most common neurologic abnormality responsible for long-term disability in the CHD population is stroke, which is detected in $10-19 \%$ of magnetic resonance imaging scans taken within the first 2 wk postoperatively $(1,2)$.

Prior clinical neuroprotection studies in the CHD population have focused on modifications of intraoperative management strategies such as more gradual induction of cooling, optimal hemodilution during bypass, and elimination or minimization of deep hypothermic circulatory arrest. Unfortunately, the results of these investigations have led to little improvement in neurodevelopmental outcomes, and more consideration is now aimed at pharmacological approaches to neuroprotection. Given that newborn heart surgery is usually a scheduled procedure, babies with CHD undergoing surgical correction are ideal subjects for neuroprotection trials because a drug candidate can be given before, during, and after surgery. Research to identify pharmacologic approaches to reduce WM brain injury and stroke is warranted.

In the brains of immature animals, investigators have focused on the role of $\alpha$-amino-3-hydroxyl-5-methyl-4-isoxazole-propionate (AMPA) and kainate glutamate receptors in the genesis of PVL. In a hypoxic-ischemic (HI) neonatal rat model of WM injury, Follett et al. (3) demonstrated WM neuroprotection after administration of the AMPA and kainate receptor antagonist 6-nitro7-sulfamoylbenzo-(f)quinoxaline-2,3-dione (NBQx). Additional studies by Follett et al. showed that a $30 \mathrm{mg} / \mathrm{kg}$ intraperitoneal (i.p.) dose of topiramate (TPM), but not a $50 \mathrm{mg} / \mathrm{kg}$ dose, was neuroprotective against WM injury following a $\mathrm{HI}$ insult in postnatal day (P)7 Long Evans rat pups (4). Furthermore, the observed reduction in WM injury was accompanied by improved functional abilities. The second most common cause of neuropathology in the CHD population is stroke. In a neonatal rat stroke model, i.p. injections of $30 \mathrm{mg} / \mathrm{kg}$ of TPM in P7 Sprague-Dawley rats extended the neuroprotection window of delayed-onset hypothermia after carotid artery ligation (5). Unfortunately, plasma

\footnotetext{
'Department of Clinical and Experimental Pharmacology, University of Minnesota, Minneapolis, Minnesota; ${ }^{2}$ Center for Orphan Drug Research, University of Minnesota, Minneapolis, Minnesota; ${ }^{3}$ Metrum Institute, Tariffville, Connecticut; ${ }^{4}$ Division of Pediatric Critical Care Medicine, The Children's Hospital of Philadelphia, Philadelphia, Pennsylvania; ${ }^{5}$ Department of Pediatric Neurology, University of Colorado Denver, Aurora, Colorado; ${ }^{6}$ Division of Neurology, The Children's Hospital of Philadelphia, Philadelphia, Pennsylvania; ${ }^{7}$ Current affiliation: Department of Clinical Development, Upsher-Smith Laboratories, Inc., Minneapolis, Minnesota. Correspondence: Annie Clark (clar0097@umn.edu)
} 
concentrations corresponding to the administered TPM doses in both of these studies were not measured and remain unknown. This represents a serious gap in our knowledge regarding neuroprotection with TPM, given that it precludes the ability to target therapeutic plasma concentrations in studies with other animal species and human newborns.

TPM is a US Food and Drug Administration-approved medication used for the prevention of migraine headaches in adults and the treatment of partial seizures, primary generalized tonic-clonic seizures, and seizures associated with Lennox-Gastaut syndrome in adults and children older than 2 y (6). TPM has a broad range of pharmacologic activity. It blocks voltage-gated sodium channels, augments $\gamma$-aminobutyrate at certain subtypes of $\gamma$-aminobutyrate ${ }_{A}$ receptors, inhibits AMPA and kainate glutamate ionotropic receptors, and is a weak inhibitor of some isoenzymes of carbonic anhydrase (CA-II and CA-IV). There is general consensus that the neuroprotective effect of TPM is due to AMPA-kainate glutamate receptor blockage (4).

TPM is an attractive candidate to ameliorate neurodegeneration from WM injury and stroke, the two most common forms of neuropathology in the CHD population. TPM may be suitable for human newborn neuroprotection trials because it is approved by the Food and Drug Administration for the prevention of seizures in children older than $2 \mathrm{y}$ and has been safely given to infants as young as 1 mo of age (7). It has also been safely administered to neonates with $\mathrm{HI}$ encephalopathy who underwent therapeutic hypothermia $(8,9)$. In addition, in a recent report of TPM use in
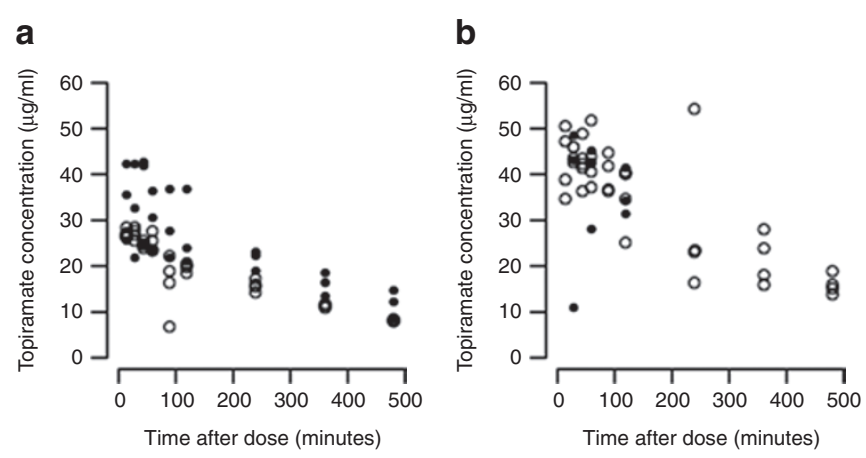

Figure 1. Observed topiramate concentrations vs. time after dose. Sprague-Dawley rats represented by open circles and Long Evans rats represented by solid circles. (a) $30 \mathrm{mg} / \mathrm{kg}$ dose; (b) $50 \mathrm{mg} / \mathrm{kg}$ dose.
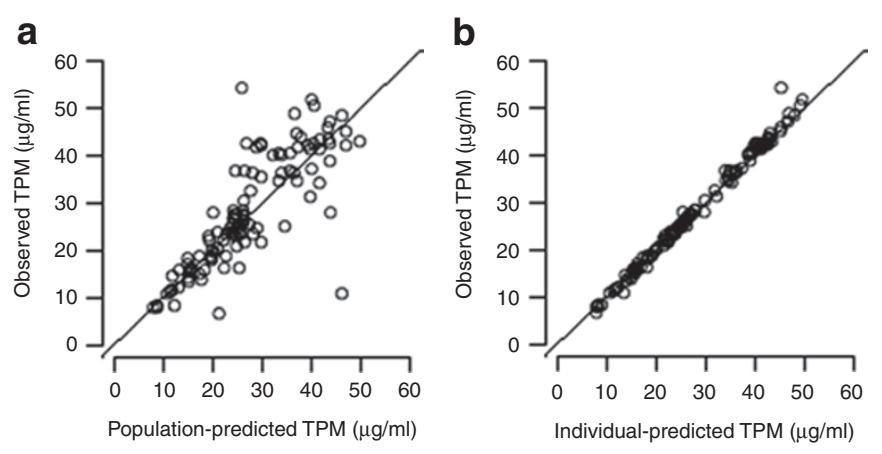

Figure 2. Diagnostic plots for the full TPM population pharmacokinetic model with covariates. (a) Population predicted vs. observed concentrations; (b) individual vs. observed concentrations. TPM, topiramate. neonates with acute symptomatic seizures that were refractory to the standard agent, none of the newborns experienced side effects resulting in discontinuation of the drug (10).

The primary aims of this study were to determine plasma concentrations associated with the administration of $30 \mathrm{mg} / \mathrm{kg}$ i.p. TPM, previously shown to ameliorate PVL and stroke in P7 rat pups and to characterize TPM pharmacokinetics (PK). Given the sparse PK sampling design (one sample per rat pup) and the covariate effects to be investigated (body size, age, and rat strain), a nonlinear mixed-effects modeling (NONMEM) approach was implemented for this analysis. This methodology allows for the pooling of PK data for simultaneous estimation of PK model parameters, between-animal variance, and covariate effects.

Results from this pilot study will help inform the design of subsequent trials intended to determine the safety, tolerability, and efficacy of TPM for neuroprotection in human neonates and infants. This approach offers a more precise way of selecting dosage regimens than the use of interspecies allometric scaling.

\section{RESULTS}

\section{Data Characteristics}

A total of 109 rat pups ranging in age from P7 to P10 were used in this experiment. Sixty-two rat pups received an i.p. TPM dose of $30 \mathrm{mg} / \mathrm{kg}$ and 47 rat pups received a $50 \mathrm{mg} / \mathrm{kg}$ dose. In this study, 72 Long Evans and 37 Sprague-Dawley rat pups weighing $19.5 \pm 3.75$ and $19.5 \pm 2.44 \mathrm{~g}$, respectively, were included. Observed plasma concentration-time profiles for 30 and $50 \mathrm{mg} / \mathrm{kg}$ TPM doses are shown in Figure 1.

\section{Population PK Modeling}

TPM disposition in rat pups was well described by a onecompartment model with first-order absorption. Diagnostic plots revealed that the model was consistent with the observed data and no systemic bias remained (Figure 2).

The change in apparent clearance $(\mathrm{CL} / F)$ and apparent volume of distribution $(V / F)$ as a function of body size was described by an allometric model (11) in which the typical value of a model parameter was described as a function of individual body weight $\left(\mathrm{WT}_{i}\right)$, normalized by a reference weight,

Table 1. Final parameter estimates from the full topiramate population pharmacokinetic model

\begin{tabular}{|c|c|c|c|c|c|}
\hline Parameter & Units & Estimate & $\%$ RSE & $90 \% \mathrm{Cl}$ & BSV \\
\hline $\mathrm{CL} / \mathrm{F}$ & $\mathrm{ml} / \mathrm{min}$ & 0.0470 & 5.59 & $(0.0398,0.0511)$ & $17.8 \%$ \\
\hline$\theta_{2}^{\text {STRAIN }}$ & & 0.691 & 9.04 & $(0.553,0.802)$ & \\
\hline$(\text { Age/9) })^{\theta 6}$ & & 1.32 & 37.6 & $(0.533,2.91)$ & \\
\hline V/F & $\mathrm{ml}$ & 22.2 & 4.91 & $(20.5,24.4)$ & $28.4 \%$ \\
\hline$\theta_{4}^{\text {STRAIN }}$ & & 0.889 & 7.90 & $(0.780,1.04)$ & \\
\hline$(\text { Age/9) })^{\theta 7}$ & & -0.787 & 61.5 & $(-1.80,-0.0554)$ & \\
\hline$k_{\mathrm{a}}$ & $\min ^{-1}$ & 0.231 & Fixed & & \\
\hline \multicolumn{6}{|c|}{ Estimates are for the typical rat pup (Sprague-Dawley, $19 \mathrm{~g}, 9 \mathrm{~d}$ of age). } \\
\hline \multicolumn{6}{|c|}{ 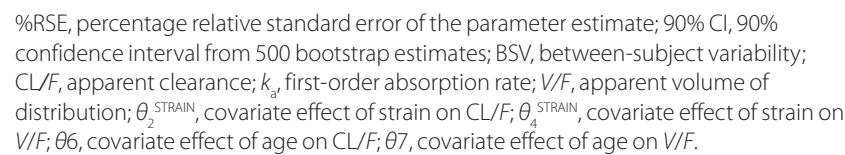 } \\
\hline
\end{tabular}


which was $19 \mathrm{~g}$. The effects of age, normalized by a reference age of $9 \mathrm{~d}$, were included in the model. The effects of strain were incorporated via a multiplicative function using an indicator variable to identify Long Evans rats (Strain =0) or SpragueDawley rats (Strain $=1$ ).

The final model was represented as

$$
\begin{aligned}
\mathrm{CL} / F & =\theta_{1} \cdot\left(\frac{\mathrm{WT}_{i}}{19 \mathrm{~g}}\right)^{0.75} \cdot \theta_{2}^{\text {Strain }} \cdot\left(\frac{\mathrm{Age}_{i}}{9 \mathrm{~d}}\right)^{\theta_{6}} \cdot e^{\eta_{\mathrm{C} / F}} \\
V / F & =\theta_{3} \cdot\left(\frac{\mathrm{WT}_{i}}{19 \mathrm{~g}}\right)^{1.0} \cdot \theta_{4}^{\text {Strain }} \cdot\left(\frac{\mathrm{Age}_{i}}{9 \mathrm{~d}}\right)^{\theta_{7}} \cdot e^{\eta_{V / F}} \\
k_{a} & =\theta_{5}
\end{aligned}
$$

$k_{\mathrm{a}}=\theta_{5}$ Parameter estimates for the full model are shown in Table 1. Parameter estimates (95\% confidence interval, CI) for

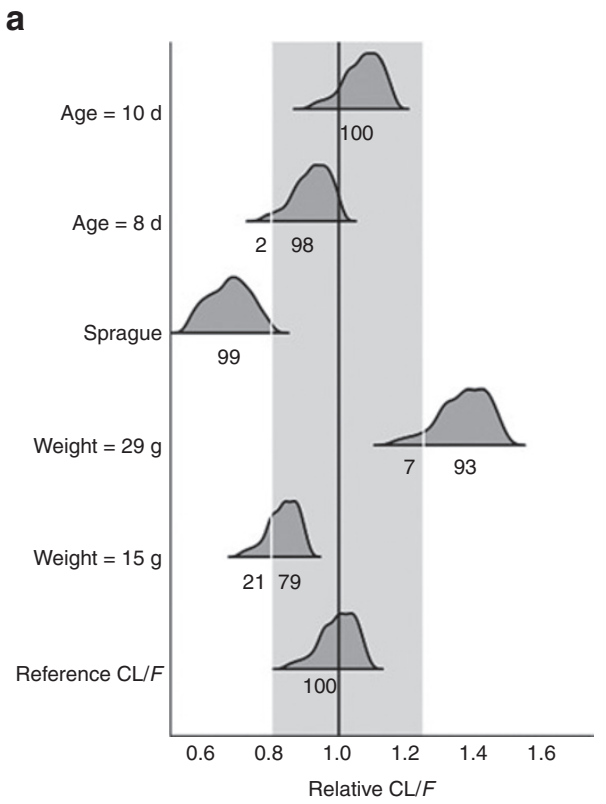

the typical rat pup (Long Evans, $19 \mathrm{~kg}, 9 \mathrm{~d})$ were 0.047 (0.0398, $0.0511) \mathrm{ml} / \mathrm{min}$ and $22.5(20.5,24.4) \mathrm{ml}$ for $\mathrm{CL} / F$ and $V / F$, respectively. Along with the parameter point estimates, measures of parameter estimation uncertainty were also obtained. These included asymptotic SE, obtained from the NONMEM \$COVARIANCE step, and 95\% CI, determined by nonparametric bootstrap. Overall, typical structural model parameters, random variance terms, and covariate effects were estimated with reasonable precision.

The CL/F in Sprague-Dawley rats was reduced by a factor of $0.691(0.553,0.802)$, whereas $V / F$ was reduced by a factor of $0.889(0.780,1.04)$ as compared with Long Evans rats. The $\mathrm{CL} / F$ and $V / F$ for Sprague-Dawley rats were $0.0324 \mathrm{ml} / \mathrm{min}$ and $19.7 \mathrm{ml}$, respectively. Although both effect estimates were well defined, with relative SE values $\leq 10.0 \%$ and relatively narrow $95 \%$ CIs, the CI for the strain effect on $V / F$ contained the null value of one. Sprague-Dawley rats had a $99 \%$ probability of

b

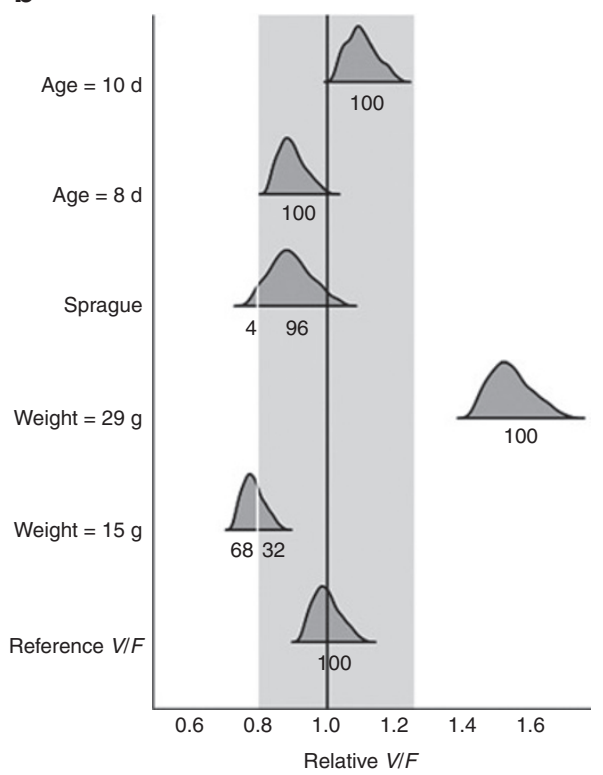

Figure 3. Relative covariate effects on topiramate pharmacokinetic parameters. The distributions (5th and 95 th percentiles) of the 1,000 nonparametric bootstrap estimates are provided as density smooths for covariate values and the reference CL/F and V/F estimates. Values for $\mathrm{CL} / F$ and $V / F$ are normalized by dividing by the named pharmacokinetics parameter's point estimate; a value of 1 represents unity or a null covariate effect. The shaded gray areas represent the $80-125 \%$ range. Numbers represent the percentage of values within each region. For weight and age, the estimates are provided at lower and upper values of the observed covariate's range. The reference rat pup was Long Evans, $19 \mathrm{~g}$, and $9 \mathrm{~d}$ of age. (a) Covariate effects on CL/F; (b) covariate effects on $V / F$. CL/F, apparent clearance; TPM, topiramate; $V / F$, apparent volume of distribution.

Table 2. Sensitivity analysis results for $k_{a}$

\begin{tabular}{lccccccccc}
\hline $\begin{array}{l}\text { Absorption } \\
t_{1 / 2}(\mathrm{~min})\end{array}$ & $k_{\mathrm{a}}\left(\mathrm{min}^{-1}\right)$ & $\mathrm{CL} / F(\mathrm{ml} / \mathrm{min})$ & $V / F(\mathrm{ml})$ & Strain on CL/F & Strain on $V / F$ & Weight on CL/F & Weight on V/F & $\omega_{C L / F}^{2}$ & $\omega_{V / F}^{2}$ \\
\hline 7 & 0.099 & 0.0486 & 21.3 & 0.694 & 0.896 & 1.30 & -0.813 & 0.0297 & 0.0907 \\
$\mathbf{5}$ & 0.139 & 0.0478 & 21.8 & 0.691 & 0.893 & 1.31 & -0.791 & 0.0309 & 0.0833 \\
$\mathbf{3}$ & $\mathbf{0 . 2 3 1}$ & $\mathbf{0 . 0 4 7 0}$ & $\mathbf{2 2 . 2}$ & $\mathbf{0 . 6 9 1}$ & $\mathbf{0 . 8 8 9}$ & $\mathbf{1 . 3 2}$ & $\mathbf{- 0 . 7 8 7}$ & $\mathbf{0 . 0 3 1 8}$ & $\mathbf{0 . 0 8 0 5}$ \\
$\mathbf{1}$ & 0.693 & 0.0466 & 22.2 & 0.691 & 0.888 & 1.32 & -0.797 & 0.0319 & 0.0807 \\
$\mathbf{0 . 5}$ & 1.386 & 0.0466 & 22.1 & 0.691 & 0.889 & 1.32 & -0.801 & 0.0319 & 0.0810 \\
$\mathbf{0 . 2 5}$ & 2.773 & 0.0465 & 22.1 & 0.691 & 0.889 & 1.32 & -0.803 & 0.0308 & 0.0811 \\
\hline
\end{tabular}

Results are presented for a range of absorption half-lives ranging from 0.25 to $7 \mathrm{~min}$. Values in bold type are from the final topiramate population pharmacokinetic model. 
Table 3. Sensitivity analysis results for residual error

\begin{tabular}{|c|c|c|c|c|c|c|c|c|c|}
\hline$\sigma^{2}$ & $\% \mathrm{CV}$ & $\mathrm{CL} / F(\mathrm{ml} / \mathrm{min})$ & $V / F(\mathrm{ml})$ & Strain on $C L / F$ & Strain on $V / F$ & Age on $\mathrm{CL} / F$ & Age on $V / F$ & $\omega_{\mathrm{CL} / \mathrm{F}}^{2}$ & $\omega_{V / F}^{2}$ \\
\hline 0.0001 & 1 & 0.0469 & 22.0 & 0.680 & 0.890 & 1.30 & -0.790 & 0.0442 & 0.0960 \\
\hline 0.0025 & 5 & 0.0470 & 22.1 & 0.684 & 0.889 & 1.30 & -0.800 & 0.0493 & 0.0935 \\
\hline 0.01 & 10 & 0.0470 & 22.2 & 0.691 & 0.889 & 1.32 & -0.787 & 0.0318 & 0.0805 \\
\hline 0.0225 & 15 & 0.0476 & 21.3 & 0.695 & 0.867 & 1.17 & -0.438 & 0.0260 & 0.0171 \\
\hline 0.0470 & 21.7 & 0.0464 & 21.2 & 0.721 & 0.829 & 1.21 & -0.372 & 0 & 0 \\
\hline
\end{tabular}

Results are presented for range of residual error values ranging from 1 to $21.7 \%$ CV.Values in bold type are from the final topiramate population pharmacokinetic model.

CV, coefficient of variation; CL/F, apparent clearance; V/F, apparent volume of distribution; $\sigma^{2}$, residual error; $\omega_{\text {CL/p }}^{2}$, between-subject variability of $C L / F$; $\omega_{V / p}^{2}$, between-subject variability of $V / F$.

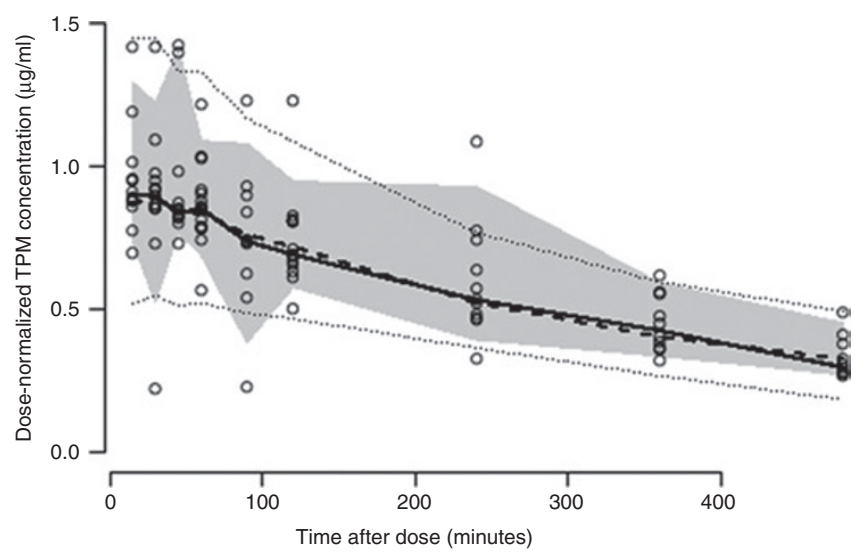

Figure 4. Visual predictive check results: observed and predicted dose-normalized topiramate concentrations vs. time after dose. Symbols represent observed topiramate concentrations (stratified by strain and dose); the solid black line is the observed median; the gray shaded area represents the observed 5 th and 95th percentiles; the dashed black line is the simulated median from 1,000 replicate trials, and the dotted black lines are the simulated 5th and 95th percentiles. TPM, topiramate.

having a $\geq 25 \%$ reduction in $\mathrm{CL} / F$ and a $4 \%$ probability for the same reduction in $V / F$ (Figure 3). This suggests that SpragueDawley rats have reduced $\mathrm{CL} / F$ and a similar $V / F$ as compared with Long Evans rats.

The effects of body weight and age on TPM PK parameters are also demonstrated in Figure 3. Relative weight effects are shown for the typical 15 and $29 \mathrm{~g}$ rat pup, representing the extremes of the data set. The probabilities for CL/F to fall outside the no-effect range for the typical 15 and $29 \mathrm{~g}$ rat were 21 and $93 \%$, respectively. For $V / F$, the probabilities were 68 and $100 \%$, respectively. This suggests that weight is an important predictor for TPM PK. Relative age effects are also shown for an 8- and 10-d-old rat pup, which represents the limits of the data set, and there was almost no probability for CL/F and $V / F$ to fall outside of the no-effect range. This demonstrates that age is not a significant predictor for TPM PK in rat pups between $8 \mathrm{~d}$ and $10 \mathrm{~d}$ of age.

Given the rapid TPM absorption after i.p. injection and a lack of plasma samples collected before 15 min after dosing, it was necessary to fix the absorption rate constant $\left(k_{a}\right)$ to a reasonable value. Therefore, $k_{a}$ was fixed to $0.231 \mathrm{~h}^{-1}$ to reflect an absorption half-life of $15 \mathrm{~min}$.

A sensitivity analysis was performed for the final model to determine the impact of the modeling assumptions (fixed residual error and $k_{a}$ ) on the resulting structural and covariate parameter estimates. The population PK model was fitted to the TPM data and values for $k_{a}$ were fixed to represent absorption half-lives of $0.25,0.5,1,5$, and $7 \mathrm{~min}$. This represented a range of plausible absorption times, ranging from 1.25 to $35 \mathrm{~min}$. The model was also fitted while fixing residual error values to 1,5 , 15 , and $22 \%$. These scenarios covered a range of possible values of minimal residual error to a maximum residual error that accounted for all of the variability in the data.

Results for the sensitivity analysis for $k_{a}$ are shown in Table 2. $\mathrm{CL} / F$ estimates ranged from 0.0465 to $0.0486 \mathrm{ml} / \mathrm{min}$, and $V / F$ ranged from 21.3 to $22.2 \mathrm{ml}$ over the range of $k_{a}$ values examined. These values represent a $<4 \%$ difference from the final model $\left(k_{a}=0.231 \mathrm{~min}^{-1}\right)$ parameter estimates. The reduction in $C L / F$ for Sprague-Dawley rats ranged from 0.691 to 0.694 , whereas the reduction in $V / F$ ranged from 0.888 to 0.896 . These estimates were $<1 \%$ different from the final model parameter estimates.

Sensitivity analysis results for residual error are shown in Table 3. CL/F estimates ranged from 0.0464 to $0.0476 \mathrm{ml} / \mathrm{min}$ and $V / F$ ranged from 21.2 to $22.2 \mathrm{ml}$, resulting in a $<4 \%$ change from the final model. The reduction in CL/ $F$ for SpragueDawley rats ranged from 0.680 to 0.721 , and the reduction in $V / F$ ranged from 0.829 to $0.890(<\sim 7 \%$ change from final model).

In Long Evans rat pups, i.p. injections of TPM 30 and $50 \mathrm{mg} /$ $\mathrm{kg}$ resulted in steady-state area under the concentration-time curve values of $202(186,239)$ and $336(310,398) \mu \mathrm{g} / \mathrm{ml} \cdot \mathrm{h}$, respectively, with average concentrations over the steady-state dosing interval of $16.8(15.5,19.9)$ and $28.1(25.8,33.2) \mu \mathrm{g} /$ $\mathrm{ml}$, respectively. The population mean predicted maximum concentration and minimum concentration $\left(C_{\min }\right)$ values were 31.3 and $7.17 \mu \mathrm{g} / \mathrm{ml}$, respectively, for the $30 \mathrm{mg} / \mathrm{kg}$ dose and 52.1 and $11.9 \mu \mathrm{g} / \mathrm{ml}$, respectively, for the $50 \mathrm{mg} / \mathrm{kg}$ dose.

In Sprague-Dawley rat pups, TPM 30 and $50 \mathrm{mg} / \mathrm{kg}$ administered i.p. resulted in steady-state area under the concentrationtime curve values of $293(252,366)$ and $488(420,609) \mu \mathrm{g} / \mathrm{ml} \cdot \mathrm{h}$, respectively, with average steady-state concentrations of 24.4 $(21.0,30.5)$ and $40.6(35.0,50.8) \mu \mathrm{g} / \mathrm{ml}$, respectively. The population mean predicted maximum concentration and $C_{\text {min }}$ values were 39.9 and $12.9 \mu \mathrm{g} / \mathrm{ml}$, respectively, for the $30 \mathrm{mg} / \mathrm{kg}$ dose and 66.5 and $21.5 \mu \mathrm{g} / \mathrm{ml}$, respectively, for the $50 \mathrm{mg} / \mathrm{kg}$ dose.

\section{Model Evaluation}

The visual predictive check revealed that the full model provided a reliable description of the data with good precision for 
a

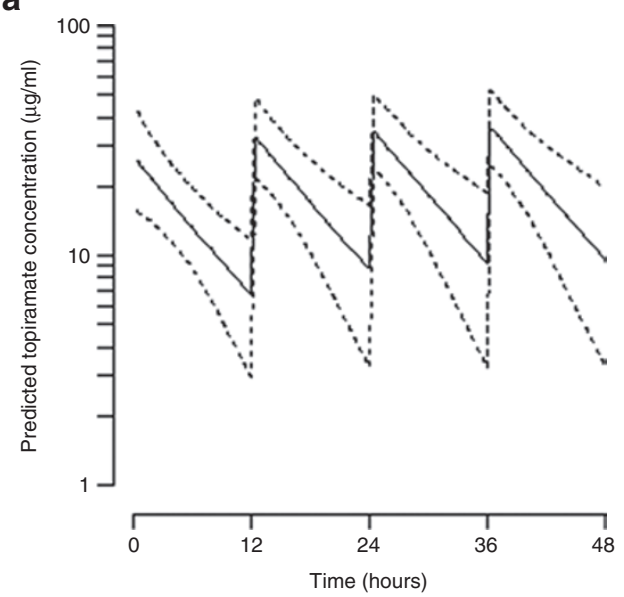

b

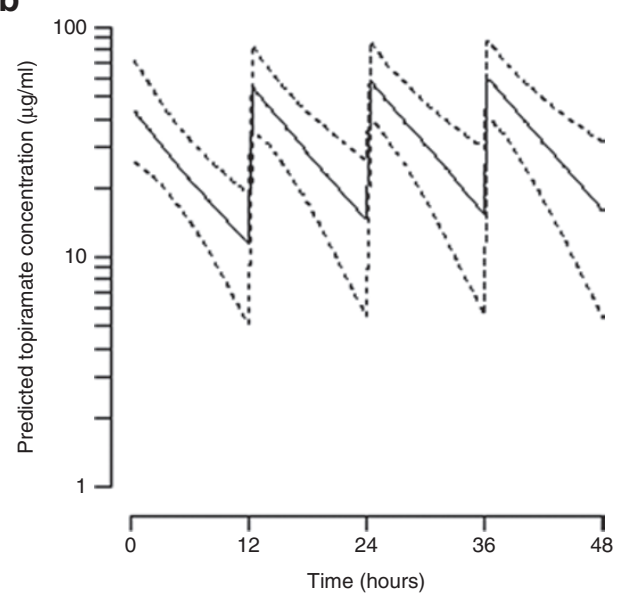

Figure 5. Simulated topiramate concentrations following four (a) $30 \mathrm{mg} /$ $\mathrm{kg}$ or (b) $50 \mathrm{mg} / \mathrm{kg}$ twice-daily doses. The solid line is the simulated median from 1,000 replicate rats, and the dashed lines are the simulated 5th and 95th percentiles.

structural model and variance parameter estimates. Figure 4 shows the observed dose-normalized TPM plasma concentrations vs. time, with the observed median (black solid line) and the observed 5th and 95th percentiles (gray shaded area) overlaid. The plot also shows results from the visual predictive check as the simulated median (solid black line) and 5th and 95th percentiles (dotted black lines) overlaid. Overall, simulated distributions were similar to the observed TPM concentrations.

\section{DISCUSSION}

PVL and stroke are the most common forms of neuropathology encountered in term infants requiring newborn heart surgery for serious forms of CHD. Hypoxia-ischemia is considered to be the primary cause of PVL. Glutamate levels increase during $\mathrm{HI}$ and cause excessive glutamate receptor activation, leading to the death of oligodendroglia and their precursors (12-15). Kainate and AMPA receptor antagonists may provide clinical benefit by preventing WM injury. Neuroprotective drugs that ameliorate white matter injury and stroke and their associated morbidities are needed.
TPM, a drug approved for treatment of epilepsy and the prevention of migraine, is neuroprotective at certain doses in newborn animal models of WM injury and stroke. Among its several mechanisms of action, TPM antagonizes both AMPA and kainate receptors. In a P7 Long Evans rat pup model of HI, i.p. injection of TPM at $30 \mathrm{mg} / \mathrm{kg}$ given every $12 \mathrm{~h}$ for four doses provided the maximal protection against PVL (4). In P7 Sprague-Dawley rat pups, the same $30 \mathrm{mg} / \mathrm{kg}$ i.p. dose of TPM extended the window of neuroprotection from hypothermia after stroke induced by carotid artery ligation. Plasma TPM concentrations were not measured in either study. Consequently, the target TPM levels for future neuroprotection studies were previously unknown. To simplify the design of our study, we used healthy pups that were not subjected to HI or carotid artery ligation. The short interval between injury and PK assessment makes it unlikely that HI would significantly alter TPM clearance, although this has not been specifically investigated. Further research is needed to determine if HI would impact TPM PK.

In Long Evans rat pups, i.p. injections of TPM $30 \mathrm{mg} / \mathrm{kg}$ results in steady-state average and minimum concentrations of 16.8 and $7.17 \mu \mathrm{g} / \mathrm{ml}$, respectively. In Sprague-Dawley rat pups, i.p. administration of $30 \mathrm{mg} / \mathrm{kg}$ TPM resulted in steady-state average and minimum concentrations of 24.4 and $12.9 \mu \mathrm{g} / \mathrm{ml}$, respectively. By contrast, the recommended therapeutic trough concentrations, $C_{\text {min }}$, for TPM administered chronically for patients with epilepsy range from 5 to $25 \mu \mathrm{g} / \mathrm{ml}$ (16). Similarly, in a study of term infants with $\mathrm{HI}$ encephalopathy treated with therapeutic hypothermia, the oral administration of $5 \mathrm{mg} / \mathrm{kg} /$ day TPM resulted in a similar range of plasma levels without any apparent adverse effects $(8,9)$.

The secondary goal of this analysis was to describe TPM PK in neonatal rat pups and to estimate the effects of covariates on the variability in PK parameters. A one-compartment model with first-order absorption was used to describe the structure of the PK model. Goodness-of-fit criteria revealed that the final model was consistent with the observed data and that little systematic bias remained. The model evaluation results also provided evidence that both the fixed and random effects components of the final model were reflective of the observed data. Estimates of covariate effects were examined in the context of the magnitude of the effect and the precision of the effect estimate.

Both Sprague-Dawley and Long Evans rat pups were used in this study because the lab inadvertently initially chose the former strain, which was a different from that used by Follet et al. The latter strain was subsequently included in the study. The analysis was performed using the full data set, rather than discarding data from one strain. Our PK analytical methodology allowed us to address the strain difference and the imbalance in sample size. The use of Long Evans and Sprague-Dawley rat pups allowed neuroprotective TPM concentrations and PK to be determined for both, which have previously been used in $\mathrm{HI}$ and stroke studies, and can guide future studies in these strains.

NONMEM allowed the effects of strain, weight, and age on TPM PK to be investigated. The estimated half-lives of TPM in this study were $5.5 \mathrm{~h}$ for a typical $19 \mathrm{~g}$, 9-d-old Long Evans 


\section{Articles | Clarketal.}

pup and $7.02 \mathrm{~h}$ for a Sprague-Dawley pup. Typically, concentration-time data were obtained over three half-lives to confidently estimate the elimination rate constant. In this study, plasma concentrations were only sampled to $8 \mathrm{~h}$ after i.p. drug administration. This constraint was partially overcome by using a population modeling approach for parameter estimation. In addition, this half-life is similar to that in other published reports in rats $(17,18)$. In humans, the half-life of TPM has been reported to range from $7.6 \mathrm{~h}$ in infants to $35 \mathrm{~h}$ in neonates undergoing hypothermia $(9,19)$.

In a PK study involving 35 infants aged 1-24 mo with partial-onset seizures, $25 \mathrm{mg} / \mathrm{kg} /$ day of TPM $(12.5 \mathrm{mg} / \mathrm{kg}$ given every $12 \mathrm{~h}$ ) resulted in plasma TPM concentrations similar to those observed in rat pups given an i.p. $30 \mathrm{mg} / \mathrm{kg}$ dose (20). The mean $C_{\min }$ was $13.6 \mu \mathrm{g} / \mathrm{ml}$, and mean area under the concentration-time curve was $\sim 211 \mu \mathrm{g} / \mathrm{ml} \cdot \mathrm{h}$. Furthermore, the above-described PK study and an efficacy trial in infants of 1-24 mo provide evidence that doses up to $25 \mathrm{mg} / \mathrm{kg} /$ day are safe (7). These results set the stage for future studies designed to characterize TPM disposition in newborns.

In the study by Follett et al., rat pups were administered TPM every $12 \mathrm{~h}$ for a total of four doses (4). This regimen would result in steady-state condition in $\sim 1 \mathrm{~d}$ based on our estimated half-life of $5.5 \mathrm{~h}$. This is illustrated in our population PK simulations of 1,000 rat pups administered 30 or $50 \mathrm{mg} / \mathrm{kg}$ according to the study design described by Follett et al. (Figure 5), which indicate that no significant drug accumulation occurs. Although median TPM plasma concentrations are markedly different when comparing 30 and $50 \mathrm{mg} / \mathrm{kg}$ dose groups, there is considerable overlap in concentrations between the two dose groups when comparing the $90 \%$ percentile intervals.

Our results indicate that average steady-state TPM concentrations of $\sim 16.8 \mu \mathrm{g} / \mathrm{ml}$ are associated with doses shown to be neuroprotective in Long Evans rat pup models of PVL induced by HI. In Sprague-Dawley rat pups, average steady-state TPM concentrations of $24.4 \mu \mathrm{g} / \mathrm{ml}$ result from doses shown to protect against stroke induced by carotid artery ligation. Future studies of TPM in other laboratory animals should use dosing regimens that attain concentrations in this range to verify that these levels are neuroprotective. Furthermore, the observation that concentrations determined in this study are similar to those used in the chronic treatment of epilepsy with TPM increases the likelihood that TPM will be safe and tolerable when prescribed as a neuroprotective agent in neonates. Nonetheless, caution in translating our results to human newborns is warranted. The pathophysiology of PVL and stroke in the human CHD population may differ from the experimentally induced brain injury in rat pups, which could affect drug response. Similarly, transport of TPM into the central nervous system of human newborns could also differ from that which occurs in rat pups. Further research is needed to determine if neuroprotective TPM concentrations in rat pups translate to human neonates.

Hypothermia during the procedure is used in many infants requiring surgical correction of CHD. It is known to alter TPM $\mathrm{PK}$ in neonates given the drug following hypoxic-ischemic encephalopathy (9). In infants treated with hypothermia, TPM maximum concentration, $C_{\text {min }}$, half-life, average concentration, and area under the concentration-time curve were higher, time of maximal concentration was delayed, and apparent total body clearance was lower as compared with normothermic infants (9). The goal of the current study was to determine TPM concentrations associated with neuroprotection in rat pups, not to determine TPM dosing during CHD correction. Therefore, further research is needed to investigate the effect that hypothermia has on TPM PK and the dosing requirements needed to attain and maintain the neuroprotective concentrations demonstrated in this study.

In future human studies, an intravenous formulation of TPM would improve the treatment of infants with CHD undergoing surgical repair. Without an intravenous formulation, treatment with oral TPM requires placement of a gastrointestinal tube. Because oral TPM solution is not commercially available, it must be extemporaneously compounded with uncertain stability. In addition, infants with CHD may have reduced gastrointestinal motility. As a result of these factors, consistent absorption cannot be assured. Therefore, an intravenous formulation of TPM would greatly facilitate appropriate treatment. Given that the absorption of TPM following i.p. dosing is rapid, the PK of TPM following intravenous dosing is likely to be similar to that following i.p. administration. In addition, the target neuroprotective concentrations should be independent of the route of administration.

Our long-term research goal was to understand, develop, and refine dosing strategies for infants with CHD who require newborn heart surgery. Our central hypothesis is that targeted TPM concentrations can ameliorate PVL and stroke in this vulnerable population. Results from this study provide target concentrations that will inform the design of TPM dosing strategies in future randomized controlled neuroprotection trials in the CHD population.

\section{METHODS}

\section{Animals}

Animal experiments were conducted in accordance with the Animal Welfare Act for the Care and Use of Laboratory Animals, and the project was approved by the University of Pennsylvania Institutional Animal Care and Use Committee. The experiments were carried out on two strains of newborn P7 to P10 rats (Long Evans and SpragueDawley). The rats were housed at Abramson Research Center rat research facility, with standard environmental conditions including 12-h light and dark cycles. Animals were allowed free access to food and water until the experiments were performed.

\section{Drug}

Pharmaceutical-grade TPM was purchased from Toronto Chemical (North York, Ontario, Canada). TPM was formulated as a $5 \mathrm{mg} / \mathrm{ml}$ solution dissolved in phosphate-buffered saline ( $\mathrm{pH}$ 7.4).

\section{Experimental Design}

Each 7-10-d-old neonatal rat pup was weighed and administered an i.p. dose of either 30 or $50 \mathrm{mg} / \mathrm{kg}$ of TPM. The animals were killed by decapitation and $1 \mathrm{ml}$ of core blood was collected at the specified time points $(15,30,60,90,120,240,360$, or $480 \mathrm{~min})$ after the injection. An average of six animals per dosing group were used for each sample time. Whole blood was centrifuged, and plasma samples were immediately frozen at $-80^{\circ} \mathrm{C}$ until analysis. 


\section{TPM Assay}

Plasma TPM concentrations were quantified with a liquid chromatography mass-spectroscopy method using electrospray ionization and negative scan mode. The analytes were separated using Zorbax LC8/ 1C18 (Agilent Technologies, Santa Clara, CA) and a mobile phase consisting of ammonium acetate buffer and methanol. Deuterated TPMd12 was added as an internal standard, and plasma samples were run with a 7-concentration standard curve (run in triplicate) and nine quality control samples (low, medium, and high, run in triplicate). The lower limit of quantification was $0.5 \mu \mathrm{g} / \mathrm{ml}$. The accuracy (\% bias) of the standards ranged from 96 to $115 \%$, and the precision or percentage coefficient of variation ranged from 0.2 to $6.7 \%$. The percentage coefficient of variation for quality controls was $<5.4 \%$.

\section{TPM Pharmacokinetic Analysis and Modeling}

Population PK analyses for repeated-measures end points were conducted via NONMEM with a qualified installation of the NONMEM software, Version VII, Level 1.2 (ICON Development Solutions, Hanover, MD). The first-order conditional estimation method with $\eta-\varepsilon$ interaction was used for all model runs (21). Goodness of fit was assessed by examining the following: visual inspection of diagnostic scatter plots (observed vs. predicted concentration, residual/weighted residual vs. predicted concentration or time, and histograms of individual random effects), precision of the parameter estimates as measured by the percentage SEM, successful minimization with at least three significant digits in parameter estimates, correlation between model parameter estimation errors $<0.95$, and changes in the estimates of interindividual and residual variability for the specified model.

Initial modeling was conducted using a one-compartment model with first-order absorption parameterized in $\mathrm{CL} / F, V / F$, and $k_{a}$, with random effect distributions for $\mathrm{CL} / F$ and $V / F$. An exponential variance model was used to describe the variability of $\mathrm{PK}$ parameters across individuals in the form: $P_{i}=\widehat{P} \exp \left(\eta_{P i}\right)$, where $P$ is the estimated parameter value for individual $i, \hat{P}$ is the typical population value (geometric mean) of the parameter, and $\eta_{\mathrm{pi}}$ represents individualspecific interindividual random effects for individual $i$ and parameter $P$ and is assumed to be distributed: $\eta \sim N\left(0, \omega^{2}\right)$ with covariances defined by the interindividual covariance matrix $\Omega$.

Several residual error models were investigated during the modelbuilding process, including proportional, additive, and combined additive and proportional. For the final model, residual error was described by proportional error model: $Y_{i j}=\widehat{Y}_{i j}\left(1+\varepsilon_{p i j}\right)$, where, $Y_{\mathrm{ij}}$ is the $j$ th measured observation (plasma TPM concentration) in individual $i, \hat{Y}_{i j}$ is the $j$ th model-predicted value (plasma TPM concentration) in individual $i$, and $\varepsilon_{\text {pij }}$ is the proportional residual random for individual $i$ and measurement $j$ and is assumed to be independently and identically distributed: $\varepsilon \sim N I D\left(0, \sigma^{2}\right)$.

Because each animal contributed only one TPM plasma concentration for the analysis, it was not possible to simultaneously estimate between-animal and residual random effects parameters due to identifiability issues. Therefore, initial model runs were conducted while fixing the residual error to a value of 0.01 to reflect the approximate magnitude of the TPM assay error.

\section{Covariate Modeling}

A covariate modeling approach emphasizing parameter estimation rather than stepwise hypothesis testing was implemented for this analysis. First, covariate-parameter relationships were identified based on scientific interest and prior knowledge of mechanistic plausibility. A covariate model was constructed with care to avoid correlation or collinearity in predictors. Population parameters, including fixed-effects parameters (covariate coefficients and structural model parameters) and random-effects parameters, were estimated. Inferences about the relevance of parameters were based on the resulting parameter estimates of the full model and measures of estimation precision. For this analysis, body weight, age, and strain (Sprague-Dawley or Long Evans) were investigated for their effects on TPM PK.

Estimates of covariate effects were examined in the context of the magnitude of the effect and the precision of the effect estimate. An effect estimate for a dichotomous covariate with a narrow CI that is completely contained within an unimportant effect size (e.g., 80-125\% of the typical value), indicates that the current data set contains sufficient information to rule out a meaningful effect of that categorical covariate. This finding would have to be viewed within the context of exposure-response relationships or target plasma concentrations in order to make a definitive determination regarding the clinical relevance of the covariate effect. A broad CI for the covariate effect, which covers effect sizes within and outside of the unimportant range, indicates that the current data set lacks the information necessary to define the clinical importance of that covariate effect. This latter scenario could be viewed as a hypothesis-generating finding, for which additional investigation would be required to elucidate the clinical importance of that covariate.

\section{Model Evaluation}

The precision of model parameters was investigated by performing a stratified nonparametric bootstrap procedure $(22,23)$. One thousand replicate data sets were generated by random sampling with replacement and were stratified by weight and strain, using the individual as the sampling unit. Population parameters for each data set were subsequently estimated for each replicate data set, and empirical 95\% CIs were constructed by observing the 2.5 th and 97.5 th quantiles of the resulting parameter distributions for those bootstrap runs with successful convergence.

The performance of the final model was also investigated via a visual predictive check to determine if the model accurately reproduced the variability in the observed data (24). Five hundred Monte Carlo simulation replicates of the original data set were generated using the final population PK model. Plots of the observed data were constructed and overlaid with the observed median and the observed 5th and 95th percentiles. In addition, the simulated median and 5th and 95th percentiles were overlaid for comparison to the observed data distribution.

\section{STATEMENT OF FINANCIAL SUPPORT}

This research was supported through the Children's Hospital of Philadelphia Endowed Chair in Pediatric Neurology Research (R.C.), The Topiramate Research Funds (R.C.), and the generous support of Mr and Mrs Stephen E. Raynes and Mr and Mrs Michael Deluca.

Disclosure: J.C. is a consultant for Allergan, Upsher-Smith Laboratories, and Lundbeck, has received a grant from Neurelis, and has a royalty agreement with Lundbeck and CyDex.

\section{REFERENCES}

1. Mahle WT, Tavani F, Zimmerman RA, et al. An MRI study of neurological injury before and after congenital heart surgery. Circulation 2002;106(12 Suppl 1):I109-14.

2. Chen J, Zimmerman RA, Jarvik GP, et al. Perioperative stroke in infants undergoing open heart operations for congenital heart disease. Ann Thorac Surg 2009;88:823-9.

3. Follett PL, Rosenberg PA, Volpe JJ, Jensen FE. NBQX attenuates excitotoxic injury in developing white matter. J Neurosci 2000;20:9235-41.

4. Follett PL, Deng W, Dai W, et al. Glutamate receptor-mediated oligodendrocyte toxicity in periventricular leukomalacia: a protective role for topiramate. J Neurosci 2004;24:4412-20.

5. Liu Y, Barks JD, Xu G, Silverstein FS. Topiramate extends the therapeutic window for hypothermia-mediated neuroprotection after stroke in neonatal rats. Stroke 2004;35:1460-5.

6 Topamax [Package Insert]. Janssen Pharmaceuticals Inc., Titusville, NJ, 2012.

7. Novotny E, Renfroe B, Yardi N, et al. Randomized trial of adjunctive topiramate therapy in infants with refractory partial seizures. Neurology 2010;74:714-20.

8. Filippi L, Poggi C, la Marca G, et al. Oral topiramate in neonates with hypoxic ischemic encephalopathy treated with hypothermia: a safety study. J Pediatr 2010;157:361-6.

9. Filippi L, la Marca G, Fiorini P, et al. Topiramate concentrations in neonates treated with prolonged whole body hypothermia for hypoxic ischemic encephalopathy. Epilepsia 2009;50:2355-61

10. Glass HC, Poulin C, Shevell MI. Topiramate for the treatment of neonatal seizures. Pediatr Neurol 2011;44:439-42. 
11. Anderson BJ, Holford NH. Mechanism-based concepts of size and maturity in pharmacokinetics. Annu Rev Pharmacol Toxicol 2008;48: 303-32.

12. Andiné P, Sandberg M, Bågenholm R, Lehmann A, Hagberg H. Intraand extracellular changes of amino acids in the cerebral cortex of the neonatal rat during hypoxic-ischemia. Brain Res Dev Brain Res 1991;64: $115-20$.

13. Benveniste H, Drejer J, Schousboe A, Diemer NH. Elevation of the extracellular concentrations of glutamate and aspartate in rat hippocampus during transient cerebral ischemia monitored by intracerebral microdialysis. J Neurochem 1984;43:1369-74.

14. Choi DW. Excitotoxic cell death. J Neurobiol 1992;23:1261-76.

15. Hagberg H, Gilland E, Diemer NH, Andiné P. Hypoxia-ischemia in the neonatal rat brain: histopathology after post-treatment with NMDA and non-NMDA receptor antagonists. Biol Neonate 1994;66: 205-13.

16. Patsalos PN, Berry DJ, Bourgeois BF, et al. Antiepileptic drugs-best practice guidelines for therapeutic drug monitoring: a position paper by the subcommission on therapeutic drug monitoring, ILAE Commission on Therapeutic Strategies. Epilepsia 2008;49:1239-76.
17 Topamax [Package Insert]. Janssen Pharmaceuticals Inc., Titusville, NJ, NDA 020505, 1996.

18. Shank RP, Gardocki JF, Streeter AJ, Maryanoff BE. An overview of the preclinical aspects of topiramate: pharmacology, pharmacokinetics, and mechanism of action. Epilepsia 2000;41:Suppl 1:S3-9.

19. Glauser TA, Miles MV, Tang P, Clark P, McGee K, Doose DR. Topiramate pharmacokinetics in infants. Epilepsia 1999;40:788-91.

20. FDA Topiramate Pediatric Clinical Pharmacology Review. (http://www. fda.gov/downloads/Drugs/DevelopmentApprovalProcess/Development Resources/ucm129621.pdf.2008).

21. NONMEM User Manual. In: 7th edn. Dublin, Ireland: ICON Development Solutions, 2010.

22. Efron B. Bootstrap Methods: Another look at the jacknife. Ann Stat 1979;7: $1-26$.

23. Parke J, Holford NH, Charles BG. A procedure for generating bootstrap samples for the validation of nonlinear mixed-effects population models. Comput Methods Programs Biomed 1999;59:19-29.

24. Post TM, Freijer JI, Ploeger BA, Danhof M. Extensions to the visual predictive check to facilitate model performance evaluation. J Pharmacokinet Pharmacodyn 2008;35:185-202. 